\title{
Improving the Identification Performance of an Industrial Process Using Multiple Neural Networks
}

\author{
E. Shafie $i^{1, *}$, H. Jazaye ri-Rad ${ }^{2}$ \\ Department of Automation and Instrumentation, Petroleum University of Technology, Ahwaz, Iran
}

\begin{abstract}
Modelling or identification of industrial plants is the first and most crucial step in their implementation process. Artificial neural networks (ANNs) as a powerful tool for modelling have been offered in recent years. Industrial processes are often so complicated that using a single neural network (SNN) is not optimal. SNNs in dealing with complex processes do not perform as required. For example the process models with th is method are not accurate enough or the dynamic characteristics of the system are not adequately represented. SNNs are generally non-robust and they are sometimes over fitted. So in this paper, we use multiple neural networks (MNNs) for modelling. Bagging and boosting are two methods employed to construct MNNs. Here, we concentrate on the use of these two methods in modelling a continuous stirred tank reactor (CSTR) and compare the results against the SNN model. Simulation results show that the use of MNNs improves the model performance.
\end{abstract}

Keywords System identification, Industrial processes,Neural networks, Bagging, Boosting, Bootstrapping

\section{Introduction}

In recent decades, artificial neural networks (ANNs) have been extensively used in numerous applications. One of the important applications of ANNs is finding patterns or tendencies in data. ANNs are well suited for prediction and forecasting requirements such as: sale forecasting, industrial process control, oil and gas industry[1-3], hand-written word recognition, target marketing, pharmaceutical industry[4], etc. In this paper, we use ANNs to identify an industrial process. One problem with ANNs is their instability. It means that small changes in the training data used to construct the model may result in a very dissimilar model[4]. Also due to the high variance of SNNs, the model may exhibit quite a different accuracy facing unseen data (validation stage)[4]. Furthermore, in numerous cases, a SNN lacks precision. Breiman[5] has shown that for unstable predictors, combining the outputs of a number of models will reduce variance and give more precise predictions.

However, it is required that the individual neural networks in aggregation should be dissimilar and there is no advantage in aggregation of the networks if they are all identical[4]. The purpose of th is paper is to identify an industrial plant. Because of the stated reasons, we will use MNNs or ensemble neural networks for identification. Thereare several different ensemble techniques, but the

* Corresponding author:

Shafiei.elham@gmail.com (E. Shafiei)

Published online at http://journal.sapub.org/ajis

Copyright (C) 2012 Scientific \& Academic Publishing. All Rights Reserved most popular ones include some elaboration of bagging[6-9], and boosting[10-18]. In this work, we parallel the use of bagging and boosting methods in modelling a chemical plant (CSTR) and co mpare the results against the corresponding SNN model.

This paper consists of the following: in sections 1 and 2 a detailed study of bagging and boosting is presented. In section 3 the desired industrial process is introduced and then the bagging and boosting algorith ms for identification are applied to this process. So me results and conclusions are presented in sections 4 and 5. Finally, references are given in section 6 .

\section{Bagging}

Bagging (an abbreviation of bootstrap aggregation) is one of the most extensively used ANN ensemble methods. The main idea in bagged neural networks is to generate a different base model instance for each bootstrap sample, and the final outputs are the average of all base model outputs for a given input[19-21]. Some of the advantages of bagging algorithm are as follows:

- Bagging reduces variance or model inconsistency over diverse data sets from a given distribution, without increasing bias, which results in a reduced overall generalization error and enhanced stability.

- The other benefit of using bagging is related to the model selection. Since bagging transforms a group of over-fitted neural networks into a better-than perfectly-fitted network, the tedious time consuming model selection is no longer required. This could even offset the 
computational overhead needed in bagging that involves training many neural networks.

- Bagging is very robust to noise.

- Parallel execution: although the boosting algorithm (discussed in the next section) has better generalization ability than the bagging algorithm, the bagging algorithm has the benefit of training ensembles independently, hence in parallel.

Presume the training dataset $T$ is composed of $N$ instances $\left(x_{1}, y_{1}\right), \ldots,\left(x_{N}, y_{N}\right)$, where $x$ and $y$ are input and output variables, respectively. It is required to acquire $B$ bootstrap datasets. As a first step, each instance in $T$ is assigned a probability of $1 / N$, and the train ing set for each of the bootstrap member $T B$ is created by sampling with replacement $N$ times from the original dataset $T$ using the above probabilities. Hence, each bootstrap datas et $T B$ may have many instances in $T$ repeated a number of times, while other instances may be omitted. Individual neural network models are then trained on each of $T B$. Therefore, for any given input vector, the bootstrap algorithm offers $B$ different outputs. The bagging estimate is then computed by determining the mean of $B$ model predictions (see Figure 1).

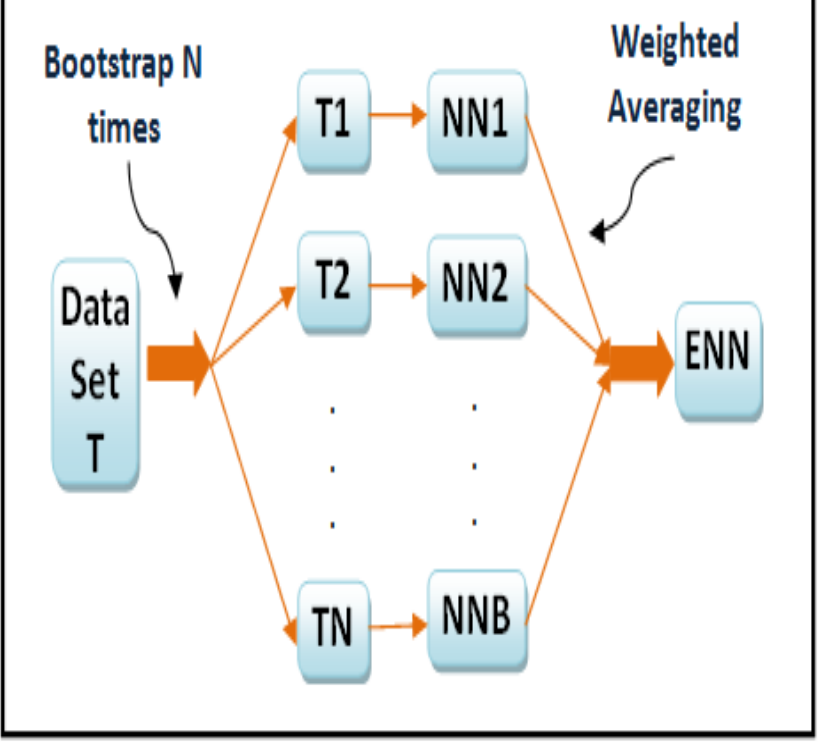

Figure 1. Bagging neural net work

The bagging algorithm can be summarized as:

1) Let $B$ be the ultimate number of predictors required (obtained by trial and error).

2) Take a training set $T=\left\{\left(x_{1}, t_{1}\right), \ldots,\left(x_{N}, t_{N}\right)\right\}$.

3) For $i=1$ to $B$ do:

3.1) Make a new training set $T B$ by sampling $N$ items evenly at rando $m$ with rep lacement fro $m$ the data set $T$.

3.2) Train an estimator $f_{i}$ with the set TB and add it to the ens emble.

4) For any new testing pattern, the bagged ensemble output is given by the equation:

$$
f_{\text {bag }}(x)=\frac{1}{B} \sum_{i=1}^{B} F_{i}(x)
$$

\section{Boosting}

Contrary to bagging, boosting dynamically tries to generate complementary learners by training the next learner on the inaccuracies of the learner in the preceding iteration. At each iteration of the algorithm the samp ling distribution depends upon the performance of the learner in the preceding iteration[22]. In spite of bagging algorith $m$ which operates in parallel,boosting algorithm is executed sequentially. In boosting, instead of a random sample of the training data, a weighted sample is used to emphasis learning on the most difficult examples.

There are numerous different versions of the boosting algorith $\mathrm{m}$ in the literature. The original boosting approach is boosting by filtering and is explained by Schapire[23]. It requires a large number of train ing data, which is not practicable in many cases. This restriction can be overcome by using another boosting algorithm known as the AdaBoost[10]. Initially the boosting algorithm was developed for binary classification problems. Then boosting algorithmssuch as AdaBoos1.M1 and AdaBoost.M2[22] were developed for multi-class cases. In order to solve regression problems, Freund and Schapire[24]extended AdaBoost.M2 and called it AdaBoost.R. It solves regression problems by converting them to classification ones.

In this paper, we use AdaBoost.R2 for identification. This method is a modification ofAdaBoost.R and is described in $[25,26]$. A description of this algorithm (shown in Figure 2) is as follows: given that the training dataset $T$ consists of $N$ instances $\left(x_{1}, y_{1}\right), \ldots,\left(x_{N}, y_{N}\right)$, where $\mathrm{x}$ and $\mathrm{y}$ are input and output variables, respectively. Initially each value in the dataset is allocated the same probability value so that each instance in the in itial dataset has an equal likelihood of being sampled in the first training set; that is, sampling distribution, $w_{i}^{t}$ at stept $=1$, is equal to $1 / N$ over all $i$, where $i=1$ to $N$.

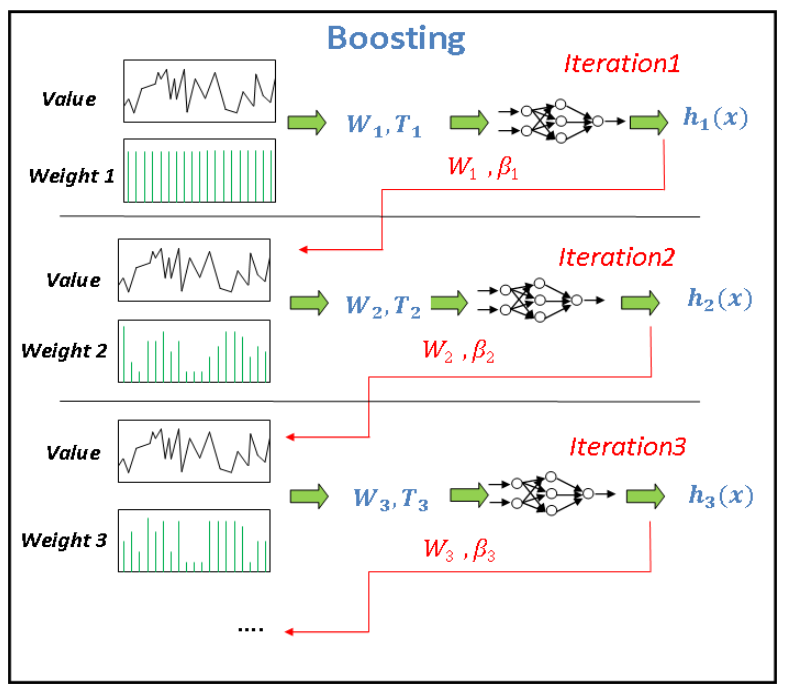

Figure 2. Boosting neural net work

The boosting algorithm can be summarized as:

1) Input the labelled target data set $T$ of theextent $N$, the maximum number of the iteration $B$, and a base algorithm 
learner. Unless otherwise stated, set the initial weight vector $w_{1}$ such that $w_{i}^{1}=1 / N$ for $1 \leq i \leq N$.

2) For $=1, \ldots, B:(B$ can be determined by a trial and error method).

2.1) Fill the new training set with the distribution $w^{t}$, and obtain a hypothesis $h(t): X \rightarrow R$.

2.2) Compute the adjusted error $e_{i}^{t}$ for each instance: $e_{i}^{t}=\left|y_{i}-h_{t}\left(x_{i}\right)\right| / D_{t}$ where $D_{t}=\max _{j=1}^{N}\left|y_{j}-h_{t}\left(x_{j}\right)\right|$.

2.3) Compute the adjusted error of $h_{t}: \varepsilon_{t}=\sum_{i=1}^{N} e_{i}^{t} w_{i}^{t}$; if $\varepsilon_{t} \geq 0.5$, stop and set $B=t-1$.

2.4) Let $\beta_{t}=\varepsilon_{t} /\left(1-\varepsilon_{t}\right)$.

2.5) Bring up to date the weight vector according to the equation:

$$
w_{i}^{t+1}=w_{i}^{t} \beta_{t}^{1-e_{i}^{t}} / Z_{t},
$$

( $Z_{t}$ is a normalizing constant).

3) Output the hypothes is: $h_{f}(x)=$ the weighted median of $h_{f}(x)$ for $1 \leq t \leq B$, using $\ln \left(1 / \beta_{t}\right)$ as the weight for the hypothesis $h_{t}$.

\section{Process Modelling Using Bagging and Boosting}

In this section, the bagging and boosting (Adaboost.R2) algorith ms are used to identify a CSTR. Since, this paper is aimed at a black box model of the process, we need to collect data directly from the process or generate it by using simulation. For this purpose, data is taken from the Daisy website[27] which is an identification database.

The process is a CSTR where the reaction is exothermic and the concentration is controlled by adjusting the coolant. The input variable is the coolant flow rate (lit/ $\mathrm{min}$ ) and the output variable is the product concentration (mol/lit). Sampling time is 0.1 minutes and the number of samples is 7500. Th is data is in the form of a $(3 \times 7500)$ matrix. The first column of this matrix consists of time-steps, the second and third columns are input (coolant flow rate) and output (concentration) variables, respectively[27]. The input and output variables are shown in Figures 3 and 4. As shown in Figure 3 , the input is constantly changing. So, the system is operated in dynamic mode.

In this paper, the bootstrap method is used to form subsystems in the bagging algorith $\mathrm{m}$. The bootstrap procedure involves choosing randomsamples with replacement fro $m a$ data set and analysing each sample the same way.

For the bagging algorithm, we use ten independent networks $(B=10)$ and weights of each network are initiated randomly. Each network is composed of one hidden layer and the activation function of output layer is linear (purelin). However, the number of hidden neurons, their activation functions and learning algorithms are different. These specifications are listed in Table 1. For the hidden layer hyperbolic tangent sigmoid (tansig) and log sig mod (logsig) transfer functions are employed. For thenetwork training, Levenberg-Marquardt backpropagation (trainlm) and Bey- sian regulation backpropagation (trainbr) are used. Each individual network is trained for 10 iterations.

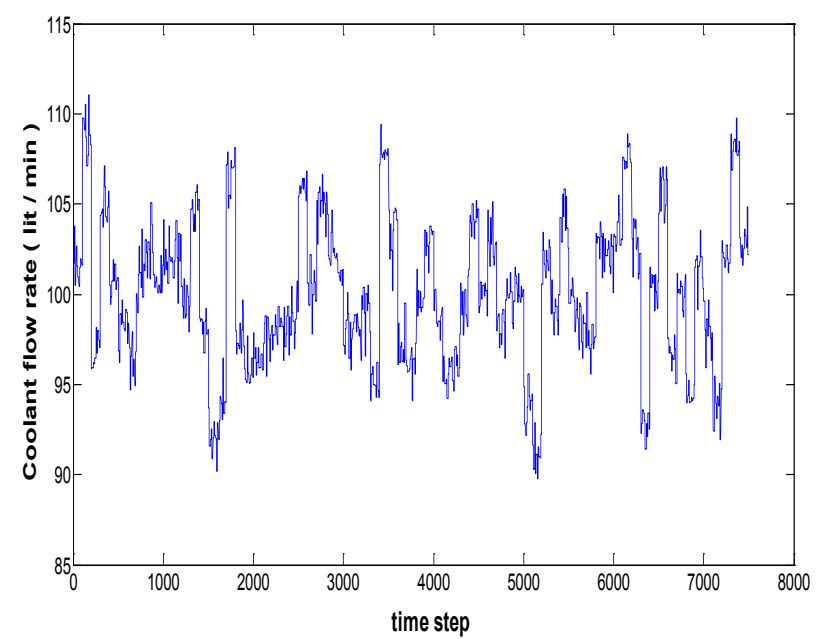

Figure 3. Input variable (coolant flow rate)

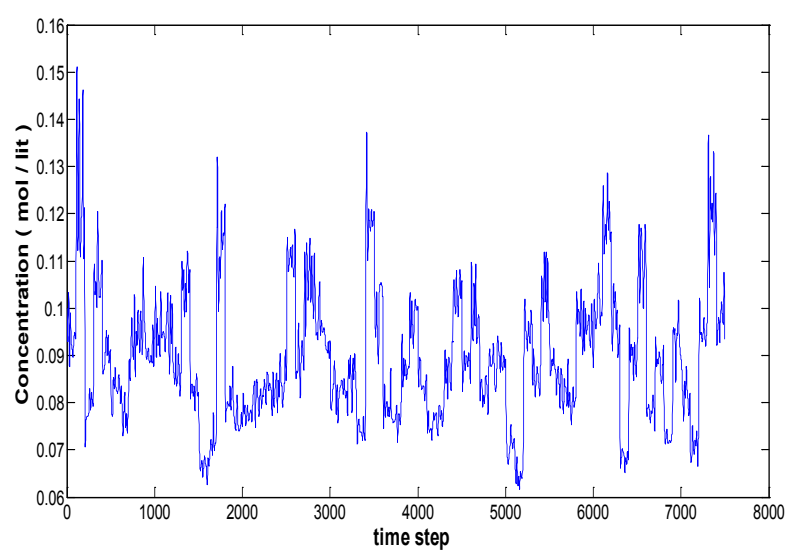

Figure 4. Output variable (concentration)

As mentioned before, the system is in dynamic mode, so the previous inputs and outputs affectthe output $y(t)$ in the present time. In this case, the inputs to the network are the inputs in the present time $u(t)$, the previous input $u(t-$ 1 , and the previous output $y(t-1)$. So, the neural network consists of three inputs $(u(t), u(t-1)$ and $y(t-1))$, and one output $y(t)$.

To determine the final predicted output of the trained ensemble, an average is taken over the predictions from individual networks. The results and conclusions are given in the following sections.

For system identification using the Adaboost.R2 algorith m, the number of iterations $B$ should be determined. We select $B$ to be equal to 20 . All sequential networks are trained using the Levenberg-Marquardtback-propagational gorithm. Each network consists of two layers. Activation functions in the output layer are "purelin" and in the hidden layer are "tansig"or "logsig". The stopping goal for the single and every individual network is the mean squared error $(\mathrm{mse}=0.000001)$. As described in the previous section, the lag space is equal to one; therefore, the number of sampled data points employed for modelling is 7499 . So, the input-output data points are in the form of $[(u(t), u(t-$ 
1), $y(t-1) ; y(t))]$. At the first iteration, all of the data points have equal chance to be selected. So, the probability of each data point to be elected is equal to $1 /_{N}=1 / 7499=$ $1.333 \times 10^{\wedge}(-4)$. Using the Adaboost.R2 algorithm described in the previous section, we will see that the value of $\varepsilon_{t}$ prior to the final iteration is less than the threshold value of 0.5 , however at the final iteration $\varepsilon_{t}>0.5$. Hence, at this point the algorithm stops.

Table 1. Specifications of the networks used in this work

\begin{tabular}{|c|c|c|c|}
\hline network & $\begin{array}{c}\text { Number } \\
\text { ofneurons }\end{array}$ & $\begin{array}{c}\text { Activation } \\
\text { function in HL }\end{array}$ & $\begin{array}{c}\text { Training } \\
\text { algorithm }\end{array}$ \\
\hline $\mathbf{1}$ & 5 & tansig & trainlm \\
\hline $\mathbf{2}$ & 6 & tansig & trainbr \\
\hline $\mathbf{3}$ & 5 & logsig & trainlm \\
\hline $\mathbf{4}$ & 6 & logsig & trainbr \\
\hline $\mathbf{5}$ & 5 & tansig & trainlm \\
\hline $\mathbf{6}$ & 6 & tansig & trainbr \\
\hline $\mathbf{7}$ & 5 & logsig & trainlm \\
\hline $\mathbf{8}$ & 6 & logsig & trainbr \\
\hline $\mathbf{9}$ & 5 & tansig & trainlm \\
\hline $\mathbf{1 0}$ & 6 & tansig & trainbr \\
\hline
\end{tabular}

\section{Results}

Table 2. Performance measures of the SNNs and MNN (thebagging neural net work)

\begin{tabular}{|c|c|c|c|}
\hline $\begin{array}{c}\text { Individual } \\
\text { network }\end{array}$ & $\begin{array}{c}\text { Standard } \\
\text { De viation }\end{array}$ & $\begin{array}{c}\text { Sum Absolute } \\
\text { Errors }\end{array}$ & R-Squared \\
\hline $\mathbf{1}$ & 0.000747 & 0.320194 & 0.99855 \\
\hline $\mathbf{2}$ & 0.000817 & 0.333045 & 0.99829 \\
\hline $\mathbf{3}$ & 0.000850 & 0.509012 & 0.99837 \\
\hline $\mathbf{4}$ & 0.000806 & 0.354326 & 0.99849 \\
\hline $\mathbf{5}$ & 0.000767 & 0.364739 & 0.99858 \\
\hline $\mathbf{6}$ & 0.000996 & 0.430697 & 0.99791 \\
\hline $\mathbf{7}$ & 0.000800 & 0.330078 & 0.99839 \\
\hline $\mathbf{8}$ & 0.000794 & 0.410069 & 0.99859 \\
\hline $\mathbf{9}$ & 0.000915 & 0.413668 & 0.99790 \\
\hline $\mathbf{1 0}$ & 0.000923 & 0.404273 & 0.99783 \\
\hline SNN & $\mathbf{0 . 0 0 1 0 4 0}$ & $\mathbf{0 . 4 1 1 6 2 2}$ & $\mathbf{0 . 9 9 7 3 2}$ \\
\hline $\mathbf{M N N}$ & $\mathbf{0 . 0 0 0 7 8 3}$ & $\mathbf{0 . 3 8 7 0 1 0}$ & $\mathbf{0 . 9 9 8 5 3}$ \\
\hline
\end{tabular}

Table 3. Comparison between single neural network and multiple neural net works (theboost ing neural net work)

\begin{tabular}{|c|c|c|c|c|}
\hline Iteration & $\begin{array}{c}\text { Standard } \\
\text { Deviation }\end{array}$ & $\begin{array}{c}\text { Sum Abso- } \\
\text { lute Errors }\end{array}$ & R-Squared & Epsilon \\
\hline 1 & 0.000929 & 5.154209 & 0.99846 & 0.100205 \\
\hline 2 & 0.000732 & 3.130475 & 0.00857 & 0.084706 \\
\hline 3 & 0.000806 & 3.463874 & 0.99825 & 0.149771 \\
\hline 4 & 0.000926 & 4.784363 & 0.99773 & 0.117025 \\
\hline 5 & 0.001108 & 4.464097 & 0.99670 & 0.041901 \\
\hline 6 & 0.000953 & 5.117671 & 0.99763 & 0.223891 \\
\hline 7 & 0.001034 & 6.263647 & 0.99723 & 0.247154 \\
\hline 8 & 0.001025 & 6.281662 & 0.99732 & 0.305718 \\
\hline 9 & 0.001224 & 7.318611 & 0.99650 & 0.412131 \\
\hline 10 & 0.001069 & 6.890501 & 0.99713 & 0.428246 \\
\hline 11 & - & - & - & 0.7356287 \\
\hline SNN & 0.000929 & 5.154209 & 0.99846 & - \\
\hline MNN & 0.000597 & 3.696289 & 0.99841 & - \\
\hline
\end{tabular}

1) The performance measures of the SNN (the complete data set is used to train the SNN) and ten individual neural networks used to construct the bagged neural network and the final MNN are shown in Table 2. As shown in this table, the accuracy of the MNN is comparable to the accuracy of the SNN. However, the variance of error in the MNN, compared to the SNN, has been significantly reduced. So, we can conclude that the bagging algorithm is success ful in reducing the variance of error. In addition, thevalues tabulated for the square of the correlation coefficients(R-Squared) indicate that the regression in the MNN is better than the SNN.

2) The performance measures of the $\mathrm{SNN}$ and eleven sequential neural networks for constructing the boosted neural network or the final MNN are shown in Table 3. Using the Adaboost.R2 algorithm, we see that the value of $\varepsilon_{t}$ prior to the eleventh iteration is less than the threshold value of 0.5 , however at the eleventh iteration $\varepsilon_{t}=$ 0.7356287 . Hence, at this point the algorithm stops. Details of all the steps and the final results are shown in this table. The second column of the table shows that using the boosting algorithm leads to reductions of both of the error variance and modeling error in the MNN when compared against the SNN. It is clear from this table that regression of the MNN is better than the SNN (R-Squared closer to 1).

\section{Conclusions}

In this paper, identification of an industrial plant (CSTR) was performed using the SNN and MNN techniques. Industrial processes can be very complex and may have highly nonlinear properties. Hence, a single neural network cannot identify industrial process es with sufficient accuracy. We used multip le neural networks instead of a single neural network. We performed modelling by employing the bagging and boosting algorithms. As shown in this work, the MNNs generated by these two algorithms outperformed the performance of the single neural network. When bagging is employed, the accuracy of the MNN is comparable to the accuracy of the SNN. However, the variance of error in the $\mathrm{MNN}$, compared to the SNN, has been significantly reduced. The boosting algorithm leads to the reductions of both of the error variance and bias of the MNN when compared against the SNN.Although the boosting algorith $m$ ensures better generalization capability than the bagging algorithm, the latter algorithm has the benefit of training the ensembles individualistically, hence in parallel.

\section{REFERENCES}

[1] Kadkhodaie A., Rezaee M., Rahimpour H., “A committee neural network for prediction of normalized oil content from well log data: An example from South Pars Gas Field, Persian Gulf', Journal of Petroleum Science and Engineering, vol. 65, no. 1-2, pp. 23-32, 2009.

[2] Siwek K., Osowski S., Szupiluk R., "Ensemble Neural 
Network for Accurate Load Forecasting in a Power System", International Journal of Applied Mathematics and Computer Science, vol. 19, no. 2, pp. 303-315, 2009.

[3] Ahmad Z., Roslin F., "Modeling of Real pH Neutralization Process Using Multiple Neural Networks (MNN) Combination", International Conference on Control, Instrumentation and Mechatronics Engineering, Malaysia, 2007.

[4] Cunningham P., Carney J., Jacob S., "Stability problems with artificial neural networks and the ensemble solution", Journal of Artificial Intelligence in Medicine, vol. 20, no. 3 , pp. 217-225, 2000.

[5] Breiman L., "Bagging predictors", Journal of Machine Learning, vol. 24, no. 2, pp. 123-140, 1996.

[6] Amanda J., Sharkey A., "Combining Artificial Neural Nets: Ensemble and Modular Multi-net Systems", Springer, New York, USA, 1999.

[7] Coelho A, Nascimento D., "On the evolutionary design of heterogeneous Bagging models", International journal of Neurocomputing, vol. 73, no. 16-18, pp. 3319-3322, 2010.

[8] Chen H., Yao X., "Regularized Negative Correlation Learning for Neural Network Ensembles", IEEE Transactions On Neural Networks, vol. 20, no. 12, pp. 1962-1979, 2009.

[9] Chandrahasan R.K., Christobel Y.A., Sridhar U.R, Arockiam L., An Empirical Comparison of Boosting and BaggingAlgorithms, (IJCSIS) International Journal of Computer Science and Information Security, vol. 9, no. 11, pp. 147-152, 2011.

[10] Freund Y., Schapire R., "Experiments with a new boosting algorithm", Proceedings of the Thirteenth International Conference on Machine Learning, San Francisco, USA, 1996.

[11] Bühlmann, P., Hothorn, T., "Twin Boosting: Improved Feature Selection and Prediction", Statistics and Computing, vol. 20, no. 2, pp. 119-138, 2010.

[12] Chang W.C., Cho C.W, "Online Boosting for Vehicle Detection", IEEE Transactions on Systems, Man, and Cybernetics, Part B (Cybernetics), vol. 40, no. 3, pp. 892-902, 2010.

[13] Dimitrakakis C., Bengio S., "Phoneme and Sentence-Level Ensembles for Speech Recognition", EURASIP Journal on Audio, Speech, and Music Processing,vol. 2011, no.1, 2011.

[14] Gao C., Sang N., Tang Q., "On Selection and Combination of Weak Learners in AdaBoost", Pattern Recognition Letters, vol. 31, no. 9, pp. 991-1001, 2010.
[15] Landesa-Vázquez I., Alba-Castro J.L., "Shedding Light on the Asymmetric Learning Capability of AdaBoost", Pattern Recognition Letters, vol. 33, no. 3, pp. 247-255, 2012.

[16] Padmaja P., Jyothi P.N., Shilpa D., "Comparative Study of Network Boosting with AdaBoost and Bagging", International Journal of Engineering Science and Technology vol. 2, no. 8, pp. 3447-3450, 2010.

[17] Shen C., Li H., "On the Dual Formulation of Boosting Algorithms", IEEE Transactions on Pattern Analysis and Machine Intelligence, vol. 32, no. 12, pp. 2216-2231, 2010.

[18] Tran T.P, Cao L., Tran D., Nguyen C.D, "Novel Intrusion Detection using Probabilistic Neural Network and Adaptive Boosting", (IJCSIS) International Journal of Computer Science and Information Security, vol. 6, No. 1, pp. 83-91, 2009.

[19] Barutcoglu Z., Alpaydin E., "A Comparison of Model Aggregation Methods for Regression", Proceedings of the 13th joint international conference on Artificial neural networks and neural information processing, Istanbul, Turkey, 2003.

[20] Ahmad Z., Zhang J., "A Comparison of Different Methods for Combining Multiple Neural Networks Models", Proceeding of the International Joint Conference on Neural Networks, Berlin, Germany, 2002.

[21] Rashid T., “A Heterogeneous Ensemble Network Using Machine Learning Techniques", International Journal of Computer Science and Network Security, vol. 9, no. 8, pp. 335-339, 2009.

[22] Solomatine D. P., Shrestha D. L., “AdaBoost.RT: a Boosting Algorithm for Regression Problems", Proceedings on International Joint Conference, Budapest Hungary, 25-29 July 2004.

[23] Schapire R., "The Strength of Weak Learn ability", Journal of Machine Learning, vol. 5, no. 2, pp. 197-227,1990.

[24] Freund Y., Schapire R., "A decision-theoreticgeneralisation of on-line learning and an application of boosting", Journal of Computer and System Sciences, vol. 55, no, 1, pp. 119-139, 1997.

[25] Drucker H., "Improving regressor using boosting”, Proceedings of the 14th International Conferences on Machine Learning, Nashville, USA ,1997.

[26] Bailly K., Milgram M., "Boosting feature selection for Neural Networks based regression", International journal of Neural Networks, vol. 22, no. 5-6, pp. 748-756, 2009.

[27] http://homes.esat.kuleuven.be/ smc/daisy/ 